\title{
Space-Time NURBS-Enhanced Finite Elements for Solving the Compressible Navier-Stokes Equations
}

\author{
Michel Make*, Norbert Hosters, Stefanie Elgeti and Marek Behr \\ Chair for Computational Analysis of Technical Systems (CATS) \\ RWTH Aachen University \\ Schinkelstrasse 2, 52062 Aachen, Germany \\ web page: http://www.cats.rwth-aachen.de/ \\ * email: make@cats.rwth-aachen.de
}

\begin{abstract}
Geometries in engineering applications are commonly designed with the use of Computer-AidedDesign (CAD) tools. These tools typically utilize Non-Uniform Rational B-Splines (NURBS) to accurately represent complex geometries. Isogeometric analysis (IGA) [1] can directly be applied to such representations. The analysis of fluid flow, however, commonly involves complex threedimensional flow domains. Parametrizing such domains by means of closed volume splines can be challenging and is still an ongoing topic in the IGA community.

An alternative approach was proposed in [2] and further extended for space-time finite elements and free-surface flows in [3]. This approach suggests to use standard finite elements in the interior of the domain, supplemented with so-called NURBS-enhanced finite elements along domain boundaries. These enhanced elements make use of NURBS to accurately represent complex geometries. This approach allows for maintaining the proven computational efficiency of standard finite element methods, while utilizing the accurate geometric representation provided by the NURBS-enhanced elements.

In the current work, we apply this approach to supersonic flow problems. For these type of problems, accurate geometric representation can be important, especially due to the presence of shock waves and their interaction with solid walls. For this purpose, the compressible Navier-Stokes equations are solved using a Stabilized Space-Time (SST) finite element formulation supplemented with NURBS-enhanced finite elements along domain boundaries. This apporach is demonstrated by means of a set of two-dimensional test cases and results are compared against solutions obtained with a standard finite element formulation.
\end{abstract}

\section{REFERENCES}

[1] Hughes, T.J.R. Cottrell, J.A. and Bazilevs, Y. Isogeometric analysis: CAD, finite elements, NURBS, exact geometry and mesh refinement. Computer Methods in Applied Mechanics and Engineering Vol. 194, pp 4135-4195, (2005)

[2] Sevilla, R. Fernandez-Mendez, S. and Huerta, A. NURBS-Enhanced Finite Element Method (NEFEM): A Seamless Bridge Between CAD and FEM. Arch of Computat. Methods Eng., Vol. 18, pp. 441, (2011)

[3] Stavrev, A. Knechtges, P. Elgeti, S. and Huerta, A. Space-time NURBS-enhanced finite elements for free-surface flows in 2d. Int. J. Num. Meth. in Fluids, Vol. 81, pp. 426-450, (2016) 